10.2478/amb-2014-0008

\title{
HYPERTROPHIC SCARS AND KELOIDS - CONTEMPORARY CONCEPTS AND TREATMENT OPTIONS
}

\author{
Y. P. Yordanov ${ }^{1}$ and A. Shef ${ }^{2}$ \\ ${ }^{1}$ Unit of Plastic Surgery and Burns, Military Medical Academy, Sofia \\ ${ }^{2}$ Department of Dermatovenereology and Allergology, Military Medical Academy, Sofia
}

Summary. Wound reparative process after surgery, burns, injuries, and inflammatory processes results in a spectrum of scar formation ranging from nearly scarless healing to excessive fibrosis or atrophy. Scarring is considered a major medical problem that leads to aesthetic and functional sequelae. Scar tissue is clinically distinguished from normal skin by an aberrant color, rough surface texture, increased thickness (hypertrophy), occurrence of contraction, and firmness. In the last decade, the concept of wound healing kinetics has been developed to describe the delicate balance of cell activity involved in scar formation and remodeling. Hypertrophic scars and keloids are formed as a result of the process of abnormal wound healing. Despite all that has been written on improving the appearance of these types of scars, there are no definitive management protocols. The aim of the present article is to make a brief review of the basic wound healing, while focusing on medicine's latest understanding of the development and treatment of keloids and hypertrophic scars.

Key words: wound healing, scar assessment, hypertrophic scars, keloids, treatment

\section{INTRODUCTION}

carring is a normal reaction to injury. A scar in the skin may be defined as a "macroscopic disturbance of the normal structure and function of the skin architecture, resulting from the end products of a healed wound" [1]. In some cases abnormal scars can develop and they are extremely distressing to the patient. These scars can be hard to treat and satisfactory management usually relies on the use of a number of treatments either used in paral- 
lel, or in sequence. Unfortunately, unlike in other areas of medicine, there have been few advances in the management and treatment of abnormal scars over the past 20 years. Patients presenting with these scars are typically referred to plastic surgeons, dermatologists and other hospital departments, principally because they have concerns over their appearance or symptoms which are affecting their quality of life.

The present review is an attempt to clarify basic wound healing with an emphasis on the latest understanding of the development and treatment of keloids and hypertrophic scars.

\section{SKIN BASICS AND WOUND HEALING}

Skin consists of two main layers, the outer epidermis and the underlying dermis [2]. Specialized epidermal structures such as hair follicles, sebaceous and sweat glands, lie within the dermis without interrupting the epidermo-dermal junction. Beneath the dermis lies the supporting hypodermis or subcutaneous fatty tissue. It contains nerve fibres and sensory organs, blood vessels supplying the skin, hair follicles and lymphatics. The epidermis has five cellular strata. Cells of the basal cell layer continually divide. Older cells are displaced towards the surface where they are eventually shed. A gradual maturation process transforms the round, nucleated cells of the basal layer into the flattened, keratin-rich squamous cells found on the outer surface of the epidermis.

\section{WOUND HEALING AND SCAR FORMATION}

The normal mammalian response to a break in cutaneous integrity occurs in three overlapping but biologically distinct phases: inflammatory, proliferative, and remodeling (Fig. 1) [3, 4]. Following the initial injury, there is an initial inflammatory phase during which a hemostasis occurs and an acute inflammatory infiltrate ensues and aims to remove devitalized tissue and prevent invasive infection. Next, there is a proliferative phase during which the balance between scar formation and tissue regeneration occurs. Usually, scar formation predominates, although in fetal wound healing an impressive amount of regeneration is possible [5]. Finally, the longest and least understood phase of wound healing occurs in the remodeling phase, whose main purpose is to maximize the strength and structural integrity of the wound. The key points of each phase are discussed below. 


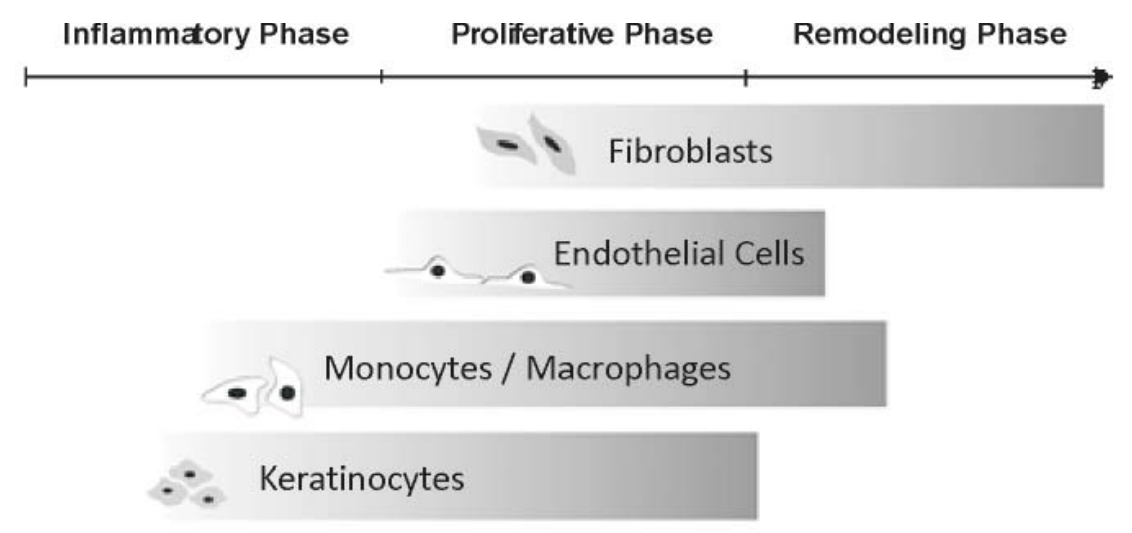

\section{(5) Neutrophils}

\section{$\approx$ Platelets}

ODays 5Days 10Days 20Days 25Days

Fig. 1. Timing of the three phases of healing process in adult cutaneous wound and the characteristic cells that are seen in the healing wound at these time points

\section{INFLAMMATORY PHASE}

Inflammation is the first stage of wound healing which starts with hemostasis [6]. At the injury site, lacerated vessels immediately constrict. Thromboplastic tissue products, predominantly from the subendothelium, are exposed. Platelets aggregate and form the initial hemostatic plug. The coagulation and complement cascades are initiated. The intrinsic and extrinsic coagulation pathways lead to activation of prothrombin to thrombin, which converts fibrinogen to fibrin, which is subsequently polymerized into a stable clot. As thrombus is formed, hemostasis in the wound is achieved. The aggregated platelets degranulate, releasing potent chemo-attractants for inflammatory cells, activation factors for local fibroblasts and endothelial cells, and vasoconstrictors. After hemostasis, local vessels dilate secondary to the effects of the coagulation and complement cascades, and to the action of different factors such as bradykinin, C3a and C5a anaphylatoxins, and histamine and leukotrienes, C4 and D4 from mast cells. The local endothelial cells then break cell-cell contact and increase permeability, which enhances the margination of inflammatory cells into the wound site. Neutrophils are the first white blood cells that infiltrate the wound and which scavenges cellular debris, foreign bodies, and bacteria [7]. Activated com- 
plement fragments aid in bacterial killing through opsonization. The neutrophil infiltrate is decreased in clean surgical wounds compared with contaminated or infected wounds. Within 2 to 3 days, the inflammatory cell population begins to shift to one of monocyte predominance. Circulating monocytes are attracted and infiltrate the wound site. These monocytes differentiate into macrophages, which not only continue to phagocytose tissue and bacterial debris, but also secrete multiple peptide growth factors [8]. These growth factors activate and attract local endothelial cells, fibroblasts, and keratinocytes to begin their respective repair functions. More than 20 different cytokines and growth factors are known to be secreted by macrophages [4, 9]. Depletion of monocytes and macrophages causes a severe alteration in wound healing with poor debridement, delayed fibroblast proliferation, and inadequate angiogenesis. The macrophage is the only inflammatory cell type that is required for normal repair, indicating its primary role in the regulation of repair [10, 11].

\section{PROLIFERATIVE PHASE}

The proliferative phase begins with degradation of the initial fibrin-platelet provisional matrix. Macrophages and the adjacent extracellular matrix (ECM) release growth factors that stimulate fibroblast activation [12, 13]. Local fibroblasts become activated and increase protein synthesis in preparation for cell division. As fibroblasts proliferate, they become the predominant cell type by 3 to 5 days in clean, non-infected wounds. After cell division and proliferation, fibroblasts begin synthesis and secretion of ECM products. The control of ECM deposition by fibroblasts is complex and partially regulated by growth factors and interactions of fibroblast cell membrane receptors with the ECM. Fibronectin and the glycosaminoglycan hyaluronic acid compose the initial wound matrix. Hyaluronic acid provides a matrix that enhances cell migration because of its large water of hydration. Adhesion glycoproteins, including fibronectin, laminin, and tenascin, are present throughout the early matrix and facilitate cell attachment and migration. Integrin receptors on cell surfaces bind to the matrix glycosaminoglycans and glycoproteins. As fibroblasts enter and populate the wound, they secrete hyaluronidase to digest the provisional hyaluronic acid-rich matrix, and larger, sulfated glycosaminoglycans are subsequently deposited. Concomitantly, new collagen is deposited by fibroblasts onto the fibronectin and glycosaminoglycan scaffold in a disorganized manner, resulting in scar formation [3$5,14]$. The major fibrillar collagens composing the ECM in skin and scar are collagen types I and III [15]. The ratio of collagen type I to type III is 4:1 in both skin and wound scar. Although type III collagen is initially deposited in relatively greater amounts in wounds, its amount is always less than type I collagen in the mature scar.

\section{GRANULATION TISSUE}

Granulation tissue is largely composed of three cell types that play critical and independent roles in granulation tissue formation: fibroblasts, macrophages, and 
endothelial cells $[3,4]$. These cells form ECM and new blood vessels, which histologically are the ingredients for granulation tissue. Granulation tissue is present in open wounds. It begins to appear in human wounds by about day 4 post-injury and is clinically characterized by its beefy-red appearance (i.e., "proud flesh"). Granulation tissue is a consequence of the rich bed of new capillary networks (neoangiogenesis) that form by endothelial cell division and migration. The directed growth of vascular endothelial cells is stimulated by platelet and activated macrophage and fibroblast products. One example is vascular endothelial growth factor, which induces migration and proliferation of endothelial cells.

\section{CONTRACTION}

Contraction is the process in which the surrounding skin is pulled circumferentially toward an open wound. This phenomenon does not occur with closed surgical incisions. The cellular mechanisms causing wound contraction are not well understood. The contractile forces are likely to be generated by myofibroblasts, which are fibroblast-like cells that contain smooth muscle $\alpha$-actin and microfilaments in their cytoplasm $[16,17]$. These cells may pull the surrounding skin toward the wound by their movement through the matrix scaffold or by intrinsic cellular forces. Wound contraction must be distinguished from contracture. Clinically, contracture is defined as tissue shortening or distortion that causes decreased joint mobility and function. Scar contracture commonly refers to decreased function in the area, whereas scar contraction refers to shortening of the scar length compared with the original wound.

\section{EPITHELIZATION}

Morphologic changes in keratinocytes at the wound margin are evident within hours after injury $[3,18]$. The epidermal cell layer thickens and marginal basal cells migrate over the wound defect. Once these keratinocytes begin migrating, they do not divide until epidermal continuity is restored. New epithelial cells for wound closure are provided by fixed basal cells in a zone near the edge of the wound. Their daughter cells flatten and migrate over the wound matrix as a sheet (epiboly). Migration of keratinocytes over the wound matrix is guided by cell adhesion glycoproteins, such as tenascin and fibronectin, which are their "railroad tracks". After the re-establishment of the epithelial layer, keratinocytes and fibroblasts secrete laminin and type IV collagen to form the basement membrane [19]. The keratinocytes become columnar and divide to restore the layering of the epidermis and re-form a barrier to further contamination and moisture loss.

\section{REMODELING PHASE}

The ECM is dynamic and is constantly undergoing remodeling during repair, which can be conceptualized as the balance between synthesis, deposition, and deg- 
radation. The balance of collagen deposition and degradation is in part determined by the regulation of matrix metalloproteinase activity. Proteins called tissue inhibitors of matrix metalloproteinase specifically inactivate the matrix metalloproteinases.

Scar formation is the ultimate outcome of wound repair in children and adults. Scar has no epidermal appendages (hair follicles and sebaceous glands), and it has a collagen pattern that is distinctly different from unwounded skin. New collagen fibers secreted by fibroblasts are present as early as 3 days after wounding. As the collagen matrix forms, densely packed fibers fill the wound site. The ultimate pattern of collagen in scar is one of densely packed fibers and not the reticular pattern found in unwounded dermis.

Wound scar remodeling occurs during months to years to form a "mature" scar. The early scar appearance is red due to its dense capillary network induced at the injury site. When closure is complete, capillaries regress until relatively few remain. As the scar redness dissipates during a period of months, the true scar pigmentation becomes evident. Scars are usually hypopigmented after full maturation. However, scars can become hyperpigmented in darker-pigmented patients and in those lighter-pigmented patients whose scars receive excess sun exposure. For this reason, sun protection measures are recommended for patients with early scars on sun-exposed areas such as the scalp, face, and neck. During remodeling, wounds gradually become stronger with time. Wound tensile strength increases rapidly from 1 to 8 weeks after wounding [20]. When the healing cascade progresses normally, approximately $30 \%$ to $50 \%$ of the final strength of the wound is achieved in 42 days. It is for this reason that elective surgery patients are told to refrain from strenuous activity or heavy lifting for at least 6 weeks. This progression represents the expected course of healing. In addition, scar is brittle and less elastic than normal skin. It is readily visible because of colour, contour, and texture differences compared with unwounded skin. Although scars can be hidden well with proper surgical planning and uneventful healing, they may have aesthetically unacceptable appearances in non-elective wounds after trauma and burns and in wounds with healing problems.

\section{EXCESSIVE HEALING}

Normal wounds have "stop" signals that halt the repair process when the dermal defect is closed and epithelialization is complete $[3,5,18]$. When these signals are absent or ineffective, the repair process may continue unabated and cause excessive scar. The underlying molecular mechanisms leading to excessive repair are not well known [14].

Notwithstanding the molecular regulation of excessive scar formation, there are clinical factors that affect scar formation [21, 22]:

1. To minimize visible scar on skin, elective incisions are least noticeable when they are placed parallel to the natural lines of skin tension (Langer's lines).

2. Sharply defined and well-aligned wound edges that are approximated without tension heal with the least amount of scar. 
3. Infection or separation of the wound edges with subsequent secondary intention healing also results in more scar formation.

4. Sun protection of all wounds is recommended to prevent scar hyperpigmentation.

Hypertrophic scars and keloids are unique to humans and do not occur in animals for unknown reasons. These pathologic scar types are distinguished on the basis of their clinical characteristics (Table 1) [14, 23-27]. Hypertrophic scars are defined as scars that have not overgrown the original wound boundaries but are instead raised. They usually form secondary to excessive tensile forces across the wound and are most common in wounds across joint surfaces on the extremities but also commonly occur on the sternum and neck. Physical therapy with rangeof-motion exercises is helpful in minimizing hypertrophic scar as well as joint contracture in the extremities. Hypertrophic scar is a self-limited type of overhealing that can regress with time. Pain and itching are commonly reported. These scars generally fade as well as flatten to the surrounding skin level. Keloids are scars that overgrow the original wound edges. This clinical characteristic distinguishes keloid from hypertrophic scar. True keloid scar is not common and occurs mainly in darkly pigmented individuals with an incidence of $4.5 \%$ to $16 \%$ in the black and Hispanic populations $[27,28]$. It has a genetic predisposition with autosomal dominant features. The keloid scar continues to enlarge past the original wound boundaries and behaves like a benign skin tumor with continued slow growth. However, complete excision with primary closure of the defect results in recurrence in the majority of cases. Keloid patients may have excessive start signals or lack the appropriate stop signals for healing [24, 25]. In the latter instance, the lack of stop signals results in continued and unchecked repair. A possible mechanism of keloid formation may be the presence of persistent signals pushing fibroblasts to keep "healing" the wound site despite complete coverage of the original wound (apoptotic gene expression is decreased in keloids, suggesting persistence of activated fibroblasts). Keloids consist mainly of collagen and are relatively acellular in their central portions with fibroblasts present along their enlarging borders [14]. They do not contain a significant excess number of fibroblasts. Keloid fibroblasts respond differently than normal wound fibroblasts to growth factors found at the repair site (i.e., TGF- $\beta 2$ treatment causes a greater degree of collagen gene expression in keloid compared with normal wound fibroblasts in in vitro studies; in addition, there is a greater degree of profibrotic growth factor expression in keloids than in normal wounds) [29]. In general, both keloid and hypertrophic scar fibroblasts have an up regulation of collagen synthesis, deposition, and accumulation. It may be that keloid fibroblasts respond to a greater degree than do hypertrophic scar fibroblasts to the signals stimulating scar formation. For example, keloid fibroblasts respond to exogenous TGF- $\beta$ with a much greater increase in collagen production than do hypertrophic scar fibroblasts. 
Table 1. Differences between keloids and hypertrophic scars

\begin{tabular}{|l|l|}
\hline Hypertrophic Scars & Keloids \\
\hline Occur soon after injury or surgery. & May not develop for many months after injury or surgery. \\
\hline Usually flatten spontaneously with time. & Remain elevated and do not spontaneously resolve. \\
\hline Limited to the area of the original tissue damage. & Not confined and may overgrow wound boundary. \\
\hline Size related to the injury. & A minor injury may produce a large lesion. \\
\hline Occur with motion and tension. & Independent of motion. \\
\hline Usually occur across flexor surface, for example joints. & $\begin{array}{l}\text { Commonly occur on ear lobes, shoulders, pre-sternal skin } \\
\text { and upper back. }\end{array}$ \\
\hline May be improved with appropriate surgery. & May be worsened with surgery. \\
\hline $\begin{array}{l}\text { Few collagen fibres; nodular structures containing fibroblastic } \\
\text { cells, small vessels and collagen. }\end{array}$ & Large, thick collagen fibres in closely packed fibrils. \\
\hline
\end{tabular}

\section{TREATMENT OF HYPERTROPHIC SCARS AND KELOID}

The treatment of hypertrophic scars and keloid is characterized by the wide variety of techniques [30]. Many of these emerged as a result of their widespread use over the past 20 years, and only a minority is actually supported by prospective studies with appropriate control groups. In addition, several therapies proved to be effective in many studies with small groups of patients were found to be not so effective in larger studies with long-term follow-up [31]. Furthermore, results evaluations of different approaches to hypertrophic scars and keloids are difficult to quantify because of natural improvement of the lesions and subsequent scars over time. The treatment of skin scarring was based largely on the experience of the physician rather than on large-scale studies.

Before anti-scarring therapy is instituted, an accurate description and classification of the scar type are useful.

\section{CLINICAL CLASSIFICATION AND SCAR ASSESSMENT}

Scar classification schemes need to be as clinically relevant as possible. The classification that fits this requirement and is worldwide accepted is the one that was proposed by Mustoe et al (Table 2) [30]. In addition, several systems to classify the severity of injury have been suggested in recent years [32]. Scar assessment tools can be classified into objective and subjective measures. An objective measure is one that can provide a quantitative assessment of the scar, in terms of its physical characteristics, such as scar colour, scar thickness and volume or pliability. A subjective measure is based on an individual's evaluation of the scar, whether the individual is a patient, clinician or lay person. Subjective measures of scarring are important to ascertain the significance of any postulated scar change. In an attempt to make subjective scar assessments more "objective" and to generate quantitative data, a variety of scar assessment scales have been developed. The most widely used system is the Vancouver Scar Scale (Table 3), together with instruments for measuring and data collection sheets [33, 34]. 
Table 2. Scar classification (by International Advisory Panel on Scar Management [30])

\begin{tabular}{|l|l|}
\hline SCAR TYPE & CHARACTERISTICS \\
\hline Mature scar & A light-colored, flat scar \\
\hline Immature scar & A red, sometimes itchy or painful, and slightly elevated scar in the process of remodeling \\
& $\begin{array}{l}\text { Many of these will mature normally over time and become flat and assume a pigmentation that } \\
\text { is similar to the surrounding skin, although they can be paler or slightly darker. }\end{array}$ \\
\hline $\begin{array}{l}\text { Linear hypertrophic scar } \\
\text { (e.g. surgical or traumatic) }\end{array}$ & $\begin{array}{l}\text { A red, raised, sometimes itchy scar confined to the border of the original surgical incision } \\
\text { This usually occurs within weeks after surgery. These scars may increase in size rapidly for } \\
3 \text { to } 6 \text { months and then, after a static phase, begin to regress. They generally mature to have } \\
\text { an elevated, slightly rope-like appearance with increased width, which is variable. The full } \\
\text { maturation process may take up to 2 years. }\end{array}$ \\
\hline $\begin{array}{l}\text { Widespread hypertrophic } \\
\text { scar (e.g. burn) }\end{array}$ & \begin{tabular}{l} 
A widespread red, raised, sometimes itchy scar confined to the border of the burn injury \\
\hline Minor keloid
\end{tabular} \\
$\begin{array}{l}\text { A focally raised, itchy scar extending over normal tissue } \\
\text { This may develop up to 1 year after injury and does not regress on its own. Simple surgical } \\
\text { excision is often followed by recurrence. There may be a genetic abnormality involved in keloid } \\
\text { scarring. Typical sites include earlobes. }\end{array}$ \\
\hline $\begin{array}{l}\text { A large, raised (> 0.5 cm) scar, possibly painful or pruritic and extending over normal tissue } \\
\text { This often results after minor trauma and can continue to spread for years }\end{array}$ \\
\hline
\end{tabular}

Table 3. Vancouver Scar Scale

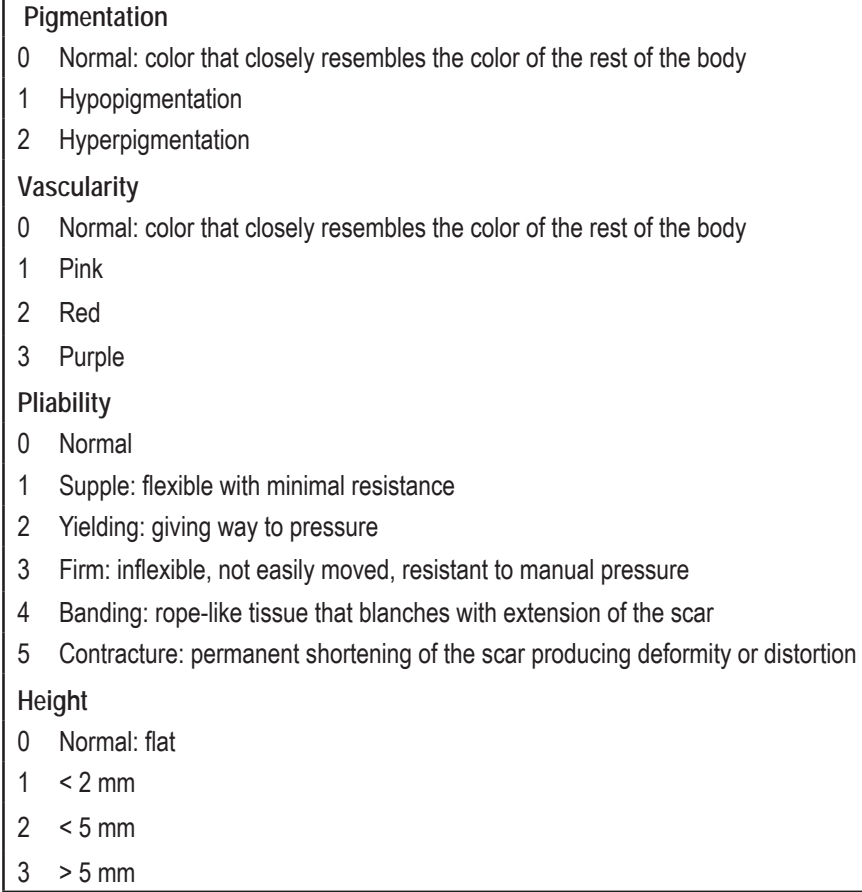




\section{PREVENTION AND TREATMENT OPTIONS}

The prevention of hypertrophic scars involves the use of a therapy with the aim of reducing the risk of pathological development. From a conceptual point of view the prevention and treatment regimens may be similar. Early diagnosis of pathological scar has a major influence on clinical outcome. The choice of therapy should be conducted according to the classification of the scar and patient's history [30].

\section{SURGERY}

Surgical excision is one of the first reported treatments for hypertrophic scars and keloids, with the first description of this technique by Druit noted in 1844 [35]. The surgical excision of hypertrophic scars or keloids is commonly used in combination with the early use of steroids after surgery and/or silicone gel sheeting [36]. Only surgical excision, in fact, is associated with high relapse rate and recurrence rates for simple surgical excision of keloids alone is reported to vary from $50 \%$ to $80 \%$ [37]. Timing of surgery can be either early or late in terms of scar maturity. It is traditional to wait for scars to be mature before performing scar revision to avoid operating on an actively hypertrophic scar when possible. This maturation period is typically 18 months, but it can be shorter or longer and may be difficult to assess. The scar should be reviewed at intervals to assess the way in which the inflammatory phase of healing is settling. As the inflammation settles, the scar and surrounding tissues become softer, more mobile, and supple. Secondary surgery before this stage of maturation is more likely to be complicated by excessive bleeding from persistently inflamed skin and subcutaneous tissue and hampered by stiffness and lack of tissue mobility, restricting the use of local flaps. The combination of surgery with intralesional steroid injections reduces the recurrence rate of keloids to less than $50 \%$, while the combination with radiation therapy reduces perioperative $10 \%$ $[31,38]$. Surgical techniques, such as $Z$ and $W$ plastic, improve the appearance and mobility of retractile scars (eg post-burn), while not suitable in the case of immature hypertrophic scars [39].

\section{INTRALESIONAL INJECTIONS OF CORTICOSTEROIDS}

The injection of corticosteroids is the treatment of choice for the treatment of keloids and the second choice for the treatment of hypertrophic scars [30, 31, 36, 40]. The mechanisms of action are complex and remain unclear [30]. Corticosteroids suppress healing by three major different mechanisms. First, inflammation is suppressed by inhibition of leukocyte and monocyte migration and phagocytosis. Second, corticosteroids are potent vasoconstrictors that may reduce the delivery of oxygen and nutrients to the wound bed. Third, and perhaps most significantly, the antimitotic effect inhibits keratinocytes and fibroblasts, slowing reepithelialization and new collagen formation. The inhibition of fibroblast proliferation by corticoste- 
roids may be dose dependent and may not be seen at lower dosages, as evidenced by tissue culture studies [41]. Insoluble triamcinolone acetonide $(10-40 \mathrm{mg} / \mathrm{ml})$, the most common corticosteroid used for the treatment of scars, may be administered alone or in combination with lidocaine to reduce the pain associated with the injection. Several treatments at once or twice a month usually are required to achieve the desired results [42, 43]. Intralesional corticosteroid injection is associated with significant injection pain, even using standard doses of triamcinolone $(40 \mathrm{mg} / \mathrm{ml})$, with up to $63 \%$ of patients experiencing some side effects [30,44]. The most common side effects of this treatment therapy are hypopigmentation, skin and subcutaneous fat atrophy, telangiectasias, rebound effects, and ineffectiveness [41-43]. After intralesional injection, linear hypopigmentation also may develop secondary to lymphogenous uptake of the corticosteroid crystals [45]. The therapeutic response rates range from 50 to $100 \%$ with a relapse rate of between 9 and $50 \%$ [46].

Topical steroid creams have been used with varying success, but it must be noted that absorption through an intact epithelium into the deep dermis is limited. A prospective, randomized study showed that topical steroids do not reduce scar formation in postburn deformities [43]. It also has been demonstrated that limited use of corticosteroids topically fails to reduce scar formation [47].

\section{SILICONE MATERIALS}

Silicones (e.g., creams, gel sheets, silastic sheets, orthosis garments) have become a very useful tool in the treatment and prevention of hypertrophic scarring, especially after burns $[48,49]$. Silicone materials are synthetic polymers based generally on a dimethyl siloxane monomer and containing a silicon-oxygen backbone, with organic groups attached directly to the silicon atom by silicon carbon bonds. Depending on the length of the polymer chain and the degree of cross-linking, the silicone can be a fluid, gel, or rubber [49,50]. Silicone is inert and does not inhibit microbial growth, but it can act as a bacterial barrier [50].

The mechanism of action of topical silicone materials on hypertrophic scars is not well understood and various mechanisms of action have been proposed [30, 43]. It has been suggested that their therapeutic effect is not because of pressure, difference in oxygen tension or temperature, or silicone leakage into the dermis [ 43 , $51,52]$. Hydration and occlusion seem to be the principal modes of SGS action, and the presence of silicone apparently is not essential to obtain beneficial clinical effects $[53,54]$. Increased skin hydration probably is responsible for a decrease in capillary activity, a reduced hyperemia, and a reduced collagen deposition [55].

\section{PRESSOTHERAPY}

Although pressure was described for the treatment of hypertrophic scars as early as the 16th century [56], and although pressure therapy often is prescribed, few controlled studies have examined its effectiveness in preventing or treating hy- 
pertrophic scarring [57]. Pressure garments have been the mainstay of hypertrophic scar treatment $[30,58]$ and currently are the standard first-line therapy for hypertrophic burn scars in many centers [30, 59-61].

To date, the working mechanism of pressure and the way pressure positively influences the development or maturation of hypertrophic scars are not fully understood, and explanations remain hypothetical [30, 62, 63]. At this writing, the exact mechanism remains unknown [43]. However, many have researched possible mechanisms of action, exploring the theories of hypoxia, biochemical changes, and cellular and collagenous influences [43,64].

Recommendations for the amount of pressure and the duration of the therapy are based merely on empirical observations [63]. Currently, many authors recommend pressures of 20 to $40 \mathrm{mmHg}$ [64]. In general, pressures that exceed $24 \mathrm{mmHg}$ are required to overcome capillary pressure [63]. This is based theoretically on the arterial capillary closing pressure of $25 \mathrm{mmHg}$ rather than on any scientific evidence. However, good clinical results (e.g., better appearance of the scar, less itching) have been reported with pressure levels as low as 5 to $15 \mathrm{mmHg}[63,65]$. Yet it also is claimed that $15 \mathrm{mmHg}$ is necessary to accelerate the maturation process and that effects of pressure below $10 \mathrm{mmHg}$ are minimal. With pressures above $40 \mathrm{mmHg}$, maceration and paresthesia may occur $[43,63]$.

\section{LASER THERAPY}

Progress in laser technology and refinements in technique have made laser therapy a preferred treatment choice for hypertrophic scars and keloids. Studies published using the $585 \mathrm{~nm}$ pulsed dye laser (PDL) have demonstrated striking improvements in scar erythema, pliability, bulk, and dysesthesia with minimal side-effects and treatment discomfort [66-68]. These observations have been substantiated by skin surface textural analysis, erythema reflectance spectrometry readings, scar height measurements, and pliability scores. Significant improvement in hypertrophic scars is generally noted within a couple of PDL treatments. Although thick keloids may require the simultaneous use of intralesional corticosteroid or 5-fluorouracil injections to enhance clinical results, the adjunctive use of intralesional corticosteroids does not significantly enhance the clinical improvement seen after PDL treatment in all but the most symptomatic or proliferative hypertrophic scars [69]. Adjacent, nonoverlapping laser pulses at fluences ranging $6.0-7.5 \mathrm{~J} / \mathrm{cm}^{2}(7 \mathrm{~mm}$ spot) or $4.5-5.5 \mathrm{~J} /$ $\mathrm{cm} 2$ (10 $\mathrm{mm}$ spot) should be applied over the entire surface of the scar [67]. Energy densities are decreased by $10 \%$ in patients with darker skin phototypes or for scars in delicate locations (e.g., the anterior chest). With PDL irradiation, the patient experiences a snapping sensation similar to that of a rubber band. Post-treatment, a mild sunburn-like sensation is produced for 15-30 minutes that is generally well-tolerated; however, some patients may require application of an ice pack. The most commonly experienced side-effect of $585 \mathrm{~nm}$ PDL treatment is post-operative purpura, which can persist for several days. Swelling of treated skin may occur immediately after 
laser irradiation, but generally subsides within 48 hours [66, 70]. Strict sun precautions should be practiced between treatment sessions in order to avoid stimulating pigment production in the treated areas. Subsequent laser sessions should be postponed until any excess pigment has resolved, so that the presence of epidermal melanin does not compromise the effectiveness of the laser. Topical bleaching agents may be used to hasten pigment resolution. Treatments are typically delivered at 6-8 week intervals; however, longer treatment intervals may be necessary for adequate healing in those patients with darker skin phototypes who develop significant postoperative hyperpigmentation [66, 68, 70].

Ultimately, an erbium-glass has been demonstrated to have a good result in scar treatment and prevention [71] but this laser is still better applied for other indications [72].

\section{RADIOTHERAPY}

Radiotherapy was used alone and in combination with surgery for the treatment of hypertrophic scars and keloids but its use as monotherapy remains controversial [30]. Although primary radiotherapy has been reserved primarily for cases of unresectable keloids, low-dose fractionated radiotherapy as an adjuvant to surgical excision may be safe and efficacious. Various regimens have been described and appear to be well tolerated [73-75]. It is reported that best results can be achieved with 1,500 to 2,000 rads (15-20 Gy) over five or six sessions in the early postoperative period [30]. The response to radiotherapy alone varies from 10 to $94 \%$ with a recurrence rate of keloids between 50 and $100 \%$. The best results were obtained with $15 \mathrm{~Gy}$ divided into 5 or 6 sessions, carried out early after surgery [76]. Some authors developed new protocols using 10, 15, or $20 \mathrm{~Gy}$, depending on the site [43]. Keloid and intractable hypertrophic scar sites with a high risk of recurrence should be treated with $20 \mathrm{~Gy}$ in 4 fractions over 4 days and that earlobe should be treated with $10 \mathrm{~Gy}$ in 2 fractions over 2 days [77]. The response to radiotherapy after surgery varies from 25 to $100 \%$ [43]. Radiotherapy, however, is difficult to evaluate because most studies are retrospective, do not define the term "recurrence", and use a variety of radiation techniques with varying follow-up periods ranging from 6 to 24 months [30]. Nevertheless, although the risk of malignant change has always been exaggerated, and although reports of carcinogenesis several years after the procedure are mainly anecdotal, this treatment method remains controversial despite the fact that there are no current reports of malignant tumors being induced by electron beam irradiation up to 128 months after irradiation [77]. The use of potentially harmful radiation therapy to treat benign lesions may be ill advised and probably cannot be justified [30, 43].

\section{CRYOTHERAPY}

Contact or spray cryosurgery with liquid nitrogen can yield significant improvement or even complete regression of hypertrophic scars and keloids $[30,73,78]$. 
Cryotherapy, when used alone, causes a flattening of keloids in $51-74 \%$ of cases [30]. However, it suffers from significant side effects such as delayed healing, permanent hypopigmentation, hyperpigmentation, atrophy, and pain [30, 79]. Therefore, this treatment is limited to small scars.

\section{OTHER THERAPIES}

Interferon $(\alpha, \beta, \gamma)$. They increase the degradation of collagen. The injection of these drugs is painful and may require regional anesthesia [30, 43, 80, 81].

Intralesional 5-fluorouracil. Several studies have confirmed that 5-FU is effective in the treatment of keloid scars [43, 80,82$]$. Wound ulceration and hyperpigmentation are reported complications, but both may resolve spontaneously [80]. It also has been used for the treatment of inflamed hypertrophic scars, either alone or in conjunction with intralesional steroid injection of PDL treatments [41, 43, 80]. The use of 5-FU intralesionally for the treatment of hypertrophic scars, either alone or in combination with corticosteroids and PDL therapy, appears to be both effective and safe [41, 46].

Bleomycin. Findings have shown that administration of bleomycin by intradermal injections or the multipuncture method is effective against keloid and hypertrophic scars [81,83], and intralesional multiple jet injections of bleomycin $0.1 \mathrm{ml}(1.5$ $\mathrm{IU} / \mathrm{ml}$ ) at a maximum dose of $6 \mathrm{ml}$ seems currently to be indicated as a therapy for keloids and hypertrophic scars unresponsive to intralesional steroid injection [81, $83,84]$. The number of sessions required for successful treatment of these lesions ranges from two to six [84], and complete to excellent flattening of scars may be expected for most patients [81].

Verapamil. This calcium-channel antagonist antiarrhythmic agent inhibits the synthesis/ secretion of extracellular matrix molecules, including collagen, glycosaminoglycans, and fibronectin, and increases collagenase [85]. A few sporadic trials have reported verapamil to be effective in the treatment of keloids when administered intralesionally $[43,86]$. The beneficial effect of calcium antagonists on pathologic scars in vivo is yet to be determined, although it has been suggested that surgical excision with W-plasty or skin grafting and intralesional verapamil injection may be a good alternative for the treatment of keloid [85].

Hyaluronic acid. It is a major component of the extracellular matrix. Experimental and clinical evidence suggests that it enhances wound healing and reduces the extent of scarring [32]. Preliminary data after intralesional hyaluronic acid injections indicate that softening of the scar is the first observed effect 4 to 6 weeks from the beginning of the treatment. A progressive reduction of the scar thickness follows until the 12th week [32].

Epicatechin gallate. Catechins are naturally occurring polyphenolic compounds believed to have antiinflammatory, antioxidant, and free radical scavenging properties [88]. However, future studies are still needed before epicatechin gallate can become part of standard therapy. 


\section{CONCLUSIONS}

The treatment choices should be based on evidence-based clinical outcomes and patient's individual needs. Further randomized controlled trials are needed to achieve a standardized therapeutic management of scars.

\section{REFERENCES}

1. Fergus on, M. W. et al. Scar formation: the spectral nature of fetal and adult wound repair. Plast. Reconstr. Surg., 97, 1996, № 4, 854-860.

2. Vandergriff, T. W. et P. R. Bergstresser. Anatomy and physiology. In: Bolognia JL, Jorizzo JL, Schaffer JV, eds. Dermatology- 3rd ed. - China, Elsevier-Sounders, 2013, 43-54.

3. Gurtne r, G. C. et V. W. Wong. Wound healing: normal and abnormal. In: Thorne $\mathrm{CH}$, Chund KC, Gosain AK, et al. eds. Grabb and Smith's Plastic Surgery. - Philadelphia, LWW, 2014, 13-19.

4. S in g e r, A. J. et R. A. Clark. Cutaneous wound healing. - N. Engl. J. Med., 341, 1999, № 10, 738-746.

5. Gurtne r, G. C. et al. Wound repair and regeneration. - Nature, 453, 2008, № 7193, 314-21.

6. Martin, P. et S. J. Leibovich. Inflammatory cells during wound repair: the good, the bad and the ugly. - Trends Cell Biol., 15, 2005, № 11, 599-607. Epub 2005 Oct 3.

7. S imps on, D. M. et R. Ross. The neutrophilic leukocyte in wound repair a study with antineutrophil serum. - J. Clin. Invest., 51, 1972, № 8, 2009-2023.

8. Gordon, S. et P. R. Taylor. Monocyte and macrophage heterogeneity. - Nat. Rev. Immunol., 5, 2005, № 12, 953-964.

9. We rn er, S. et R. Grose. Regulation of wound healing by growth factors and cytokines. - Physiol. Rev., 83, 2003, № 3, 835-870.

10. Le ibovich, S. J. et R. Ross. The role of the macrophage in wound repair. A study with hydrocortisone and antimacrophage serum. - Am. J. Pathol., 78, 1975, № 1, 71-100.

11. $\mathrm{He}$, L. et A. G. Marneros. Macrophages are essential for the early wound healing response and the formation of a fibrovascular scar. - Am. J. Pathol., 182, 2013, № 6, 2407-2417.

12. L o dis h, H. et al. Multicellularity: cell-cell and cell-matrix interactions. In: Lodish H, et al, eds. Molecular Cell Biology, 4th ed. New York, Scientific American Books, 1995, 1123-1200.

13. Hyne s, R. O. The extracellular matrix: not just pretty fibrils. - Science, 326, 2009, № 5957, 1216-1219.

14. A ti y e h, B. S., M. Costagliola et S. N. Hayek. Keloid or hypertrophic scar: the controversy: review of the literature. - Ann. Plast. Surg., 54, 2005, № 6, 676-680.

15. Volk, S. W. et al. Diminished type III collagen promotes myofibroblast differentiation and increases scar deposition in cutaneous wound healing. - Cells Tissues Organs, 194, 2011, № 1, 25-37.

16. Des moulière, A., C. Chaponnier et G. Gabbiani. Tissue repair, contraction, and the myofibroblast. - Wound Repair Regen, 13, 2005, № 1, 7-12.

17. Gabbiani, G. Evolution and clinical implications of the myofibroblast concept. - Cardiovasc. Res., 38, 1998, № 3, 545-548.

18. Woodley, D. T. Reepithelialization. In: Clark RAF, ed. The Molecular and Cellular Biology of Wound Repair. - New York, Plenum, 1996, 339-350.

19. Marinkovich, M. P. et al. Cellular origin of the dermal-epidermal basement membrane. - Dev. Dyn., 197, 1993, № 4, 255-267.

20. Buck, D. W. et R. D. Galiano. Wound Care. In: Thorne CH, Chund KC, Gosain AK, et al. eds. Grabb and Smith's Plastic Surgery. - Philadelphia, LWW, 2014, 20-28.

21. Th o mas, J. R. et M. Somenek. Scar revision review. - Arch. Facial. Plast. Surg., 14, 2012, № 3, 162-174. 
22. Ga ntwerker, E. A. et D. B. Hom. Skin: histology and physiology of wound healing. - Clin. Plast. Surg., 39, 2012, № 1, 85-97.

23. R u s s e II , S. B. et al. Reduced growth factor requirement of keloid-derived fibroblasts may account for tumor growth. - Proc. Natl. Acad. Sci. USA, 85, 1988, № 2, 587-591.

24. Nirodi, C. S. et al. Chemokine and chemokine receptor expression in keloid and normal fibroblasts. - Wound Repair Regen, 8, 2000, № 5, 371-382.

25. Tu a n, T. L. et L. S. Nichter. The molecular basis of keloid and hypertrophic scar formation. - Mol. Med. Today, 4, 1998, № 1, 19-24.

26. Rockwell, W. B., I. K. Cohen et H. P. Ehrlich. Keloids and hypertrophic scars: a comprehensive review. - Plast. Reconstr. Surg., 84, 1989, № 5, 827-837.

27. Rek ha, A. Keloids: a frustrating hurdle in wound healing. - Int. Wound J., 1, 2004, № 2, 145-148.

28. Har-Shai, Y., M. Amar et E. Sabo. Intralesional cryotherapy for enhancing the involution of hypertrophic scars and keloids. - Plast. Reconstr. Surg., 111, 2003, № 6, 1841-1852.

29. Ch o d o n, T. et al. Keloid-derived fibroblasts are refractory to Fas-mediated apoptosis and neutralization of autocrine transforming growth factor-beta1 can abrogate this resistance. - Am. J. Pathol., 157, 2000, № 5, 1661-1669.

30. M u s t o e, T. A. et al. International clinical recommendations on scar management. - Plast. Reconstr. Surg., 110, 2002, № 2, 560-571.

31. R e is h, R. G. et E. Eriksson. Scars: a review of emerging and currently available therapies. - Plast. Reconstr. Surg., 122, 2008, № 4, 1068-1078.

32. D u ra n i, P., D. A. McGrouther et M. W. Ferguson. Current scales for assessing human scarring: a review. - J. Plast. Reconstr. Aesthet. Surg., 62, 2009, № 6, 713-720.

33. Sulliva n, T. et al. Rating the burn scar. - J. Burn. Care Rehabil., 11, 1990, № 3, 256-260.

34. Van Zuijlen, P. P. et al. Scar assessment tools: implications for current research. - Plast. Reconstr. Surg., 109, 2002, № 3, 1108-1122.

35. Druit, R. Modern Surgery. Philadelphia, Lea and Blanchard, 1844.

36. Og awa, R. The most current algorithms for the treatment and prevention of hypertrophic scars and keloids. - Plast. Reconstr. Surg., 125, 2010, № 2, 557-568.

37. Darzi, M. A. et al. Evaluation of various methods of treating keloids and hypertrophic scars: a 10year follow-up study. - Br. J. Plast. Surg., 45, 1992, № 5, 374-379.

38. M a laker, A., F. Ellis et C. H. Paine. Keloid scars: a new method of treatment combining surgery with interstitial radiotherapy. - Clin. Radiol., 27, 1976, № 2, 179-183.

39. P é re z-B u s till o , A., B. González-Sixto et M. A. Rodríguez-Prieto. Surgical principles for achieving a functional and cosmetically acceptable scar. - Actas Dermosifiliogr., 104, 2013, № 1, 17-28.

40. Ts a o, S. S. et al. Scar management: keloid, hypertrophic, atrophic, and acne scars. - Semin. Cutan. Med. Surg., 21, 2002, № 1, 46-75.

41. Fitzpatrick, R. E. Treatment of inflamed hypertrophic scars using intralesional 5-FU. - Dermatol. Surg., 25, 1999, № 3, 224-232.

42. Manuskiatti, W. et R. E. Fitzpatrick. Treatment response of keloidal and hypertrophic sternotomy scars: comparison among intralesional corticosteroid, 5-fluorouracil, and 585-nm flashlamppumped pulsed-dye laser treatments. - Arch. Dermatol., 138, 2002, № 9, 1149-1155.

43. A tiye h, B. S. Nonsurgical management of hypertrophic scars: evidence-based therapies, standard practices, and emerging methods. - Aesthetic. Plast. Surg., 31, 2007, № 5, 468-92.

44. Sproat, J. E. et al. Hypertrophic sternal scars: silicone gel sheet versus Kenalog injection treatment. - Plast. Reconstr. Surg., 90, 1992, № 6, 988-992.

45. G e o r g e, W. M. Linear lymphatic hypopigmentation after intralesional corticosteroid injection: report of two cases. - Cutis, 64, 1999, № 1, 61-64. 
46. $\mathrm{Ni}$ ie s s e n, F. B. et al. On the nature of hypertrophic scars and keloids: a review. - Plast. Reconstr. Surg., 104, 1999, № 5, 1435-1458.

47. Widg e row, A. D. et al. New innovations in scar management. - Aesthetic. Plast. Surg., 24, 2000, № 3, 227-234.

48. Gibbons, M. et al. Experience with silastic gel sheeting in pediatric scarring. - J. Burn. Care Rehabil., 15, 1994, № 1, 69-73.

49. Van den Kerckhove, E. et al. Silicones in the rehabilitation of burns: a review and overview. - Burns, 27, 2001, № 3, 205-214.

50. Ch a n, K. Y. et al. A randomized, placebo-controlled, double-blind, prospective clinical trial of silicone gel in prevention of hypertrophic scar development in median sternotomy wound. - Plast Reconstr. Surg., 116, 2005, № 4, 1013-1020.

51. Atiy e h, B. S. et al. Improving scar quality: a prospective clinical study. - Aesthetic. Plast. Surg., 26, 2002, № 6, 470-476.

52. Musgrave, M. A. et al. The effect of silicone gel sheets on perfusion of hypertrophic burn scars. - J. Burn Care Rehabil., 23, 2002, № 3, 208-214.

53. Ricketts, C. H. et al. Cytokine mRNA changes during the treatment of hypertrophic scars with silicone and nonsilicone gel dressings. - Dermatol. Surg., 22, 1996, № 11, 955-959.

54. S a wa da, Y. et K. Sone. Hydration and occlusion treatment for hypertrophic scars and keloids. Br. J. Plast. Surg., 45, 1992, № 8, 599-603.

55. Niesse n, F. B. et al. The use of silicone occlusive sheeting (Sil-K) and silicone occlusive gel (Epiderm) in the prevention of hypertrophic scar formation. - Plast. Reconstr. Surg., 102, 1998, № 6, 1962-1972.

56. Lin are s, H. A., D. L. Larson et B. A. Willis-Galstaun. Historical notes on the use of pressure in the treatment of hypertrophic scars or keloids. - Burns, 19, 1993, № 1, 17-21.

57. B o $\mathrm{mb}$ a r o, K. M. et al. What is the prevalence of hypertrophic scarring following burns? - Burns, 29, 2003, № 4, 299-302.

58. Staley, M. J. et R. L. Richard. Use of pressure to treat hypertrophic burn scars. - Adv. Wound Care, 10, 1997, № 3, 44-46.

59. Ne delec, B. et al. Control of wound contraction. Basic and clinical features. - Hand Clin., 16, 2000, № 2, 289-302.

60. Es s e Im a n, P. C. et al. Burn rehabilitation: state of the science. - Am. J. Phys. Med. Rehabil., 85, 2006, № 4, 383-413.

61. R a y n e r, K. The use of pressure therapy to treat hypertrophic scarring. - J. Wound Care, 9, 2000, № 3, 151-153.

62. Macintyre, L. et M. Baird. Pressure garments for use in the treatment of hypertrophic scars-a review of the problems associated with their use. - Burns, 32, 2006, № 1, 10-15.

63. Van den Kerckhove, E. et al. The assessment of erythema and thickness on burn related scars during pressure garment therapy as a preventive measure for hypertrophic scarring. - Burns, 31, 2005, № 6, 696-702.

64. Giele, H. et al. Anatomical variations in pressures generated by pressure garments. - Plast. Reconstr. Surg., 101, 1998, № 2, 399-406.

65. Wa rd, R. S. Pressure therapy for the control of hypertrophic scar formation after burn injury. A history and review. - J. Burn Care Rehabil., 12, 1991, № 3, 257-262.

66. Alster, T. S. et T. O. McMeekin. Improvement of facial acne scars by the $585 \mathrm{~nm}$ flashlamppumped pulsed dye laser. - J. Am. Acad. Dermatol., 35, 1996, № 1, 79-81.

67. Alster, T. S. Improvement of erythematous and hypertrophic scars by the 585-nm flashlamppumped pulsed dye laser. - Ann. Plast. Surg., 32, 1994, № 2, 186-190. 
68. Alster, T. S. et C. M. Williams. Treatment of keloid sternotomy scars with $585 \mathrm{~nm}$ flashlamppumped pulsed-dye laser. - Lancet, 345, 1995, № 8959, 1198-1200.

69. Alster, T. Laser scar revision: comparison study of 585-nm pulsed dye laser with and without intralesional corticosteroids. - Dermatol. Surg., 29, 2003, № 1, 25-29.

70. K h a tri, K. A., D. L. Mahoney et M. J. McCartney. Laser scar revision: A review. - J. Cosmet. Laser Ther., 13, 2011, № 2, 54-62.

71. Kim, H. S. et al. Comparison of the effectiveness of nonablative fractional laser versus ablative fractional laser in thyroidectomy scar prevention: A pilot study. - J. Cosmet. Laser Ther., 14, 2012, № 2, 89-93.

72. S h e f, A. et M. Kadurina. Vascular anomalies: port wine stains and child's hemangioma. Light and laser therapy as an effective approach in their treatment. Dermatology and Venereology, 50, L, 1 [Article in Bulgarian]

73. Che n, M. A. et T. M. Davidson. Scar management: prevention and treatment strategies. - Curr. Opin. Otolaryngol. Head Neck Surg., 13, 2005, № 4, 242-247.

74. D in h, Q., M. Veness et S. Richards. Role of adjuvant radiotherapy in recurrent earlobe keloids. Australas J. Dermatol., 45, 2004, № 3, 162-166.

75. M a la ker, K. et al. Retrospective analysis of treatment of unresectable keloids with primary radiation over 25 years. - Clin. Oncol. (R Coll Radiol), 16, 2004, № 4, 290-298.

76. Og awa, R. et al. Postoperative radiation protocol for keloids and hypertrophic scars: statistical analysis of 370 sites followed for over 18 months. - Ann. Plast. Surg., 56, 2007, № 6, 688-691.

77. O gawa, R. et al. Postoperative electron-beam irradiation therapy for keloids and hypertrophic scars: retrospective study of 147 cases followed for more than 18 months. - Plast. Reconstr. Surg., 111, 2003, № 2, 547-53.

78. Har-Shai, Y., M. Amar et E. Sabo. Intralesional cryotherapy for enhancing the involution of hypertrophic scars and keloids. - Plast. Reconstr. Surg., 111, 2003, № 6, 1841-1852.

79. Rus cian i, L., G. Rossi et R. Bono. Use of cryotherapy in the treatment of keloids. - J. Dermatol. Surg. Oncol., 19, 1993, № 6, 529-534.

80. A pikian, M. et G. Goodman. Intralesional 5-fluorouracil in the treatment of keloid scars. -Australas J. Dermatol., 45, 2004, № 2, 140-143.

81. Ya m a m o to, T. Bleomycin and the skin. - Br. J. Dermatol., 155, 2006, № 5, 869-875.

82. LebwohI, M. From the literature: intralesional 5-FU in the treatment of hypertrophic scars and keloids: clinical experience. - J. Am. Acad. Dermatol., 42, 2000, № 4, 677.

83. España, A., T. Solano et E. Quintanilla. Bleomycin in the treatment of keloids and hypertrophic scars by multiple needle punctures. - Dermatol. Surg., 27, 2001, № 1, 23-27.

84. Sa ra y, Y. et A. T. Güleç. Treatment of keloids and hypertrophic scars with dermojet injections of bleomycin: a preliminary study. - Int. J. Dermatol., 44, 2005, № 9, 777-784.

85. C opcu, E., N. Sivrioglu et Y. Oztan. Combination of surgery and intralesional verapamil injection in the treatment of the keloid. - J. Burn Care Rehabil., 25, 2004, № 1, 1-7.

86. Giuglia n o, G. et al. Verapamil inhibits interleukin-6 and vascular endothelial growth factor production in primary cultures of keloid fibroblasts. - Br. J. Plast. Surg., 56, 2003, № 8, 804-809.

87. B orgognoni, L. et U. M. Reali. Intralesional hyaluronic acid treatment of pathological scars. Ann. Plast. Surg., 38, 1997, № 3, 308, 7A.

88. Ka p o or, M., R. Howard, I. Hall et I. Appleton. Effects of epicatechin gallate on wound healing and scar formation in a full thickness incisional wound healing model in rats. - Am. J. Pathol., 165, 2004, № 1, 299-307.

\section{Corresponding author:}

Yordan Petrov Yordanov, MD, PhD

Military Medical Academy

3, Sv. Georgy Sofiyski st.

$\mathrm{Bg}-1606$ Sofia

+359887560054

e-mail: yordanov_vma@abv.bg 